\title{
Baseline Wander Correction and Impulse Noise Suppression Using Cascaded Empirical Mode Decomposition and Improved Morphological Algorithm
}

\author{
Ashis Kumar Das \\ Electrical Engineering Department, National Institute of Technology, Durgapur - 713209, India. \\ Faculty of Technology, Uttar Banga Krishi Viswavidyalaya, Coochbehar- 736101, India. \\ ORCID: 0000-0002-0013-7176 \\ Suman Halder \\ Electrical Engineering Department, National Institute of Technology, Durgapur - 713209, India.
}

ORCID: 0000-0003-0219-9562

\begin{abstract}
When the cardiac impulse passes through the heart, electrical current also spreads from the heart into the adjacent tissues surrounding the heart. A small portion of the current spreads all the way to the surface of the body. If electrodes are placed on the skin on opposite sides of the heart, electrical potentials generated by the current can be recorded; the recording is known as an electrocardiogram. It is used for diagnosis of a number of cardiac diseases and disorders. But the ECG signals are often corrupted by noises and artefacts. For getting the proper information from ECG, it first must be de-noised. ECG signal is dominated mainly by high frequency impulse noises and baseline wander. These noise must be removed for better clinical assessment. In this paper, we propose an improve ECG intensification method based on empirical mode decomposition and improved morphological algorithm. The method is examined through experimental simulations on MIT-BIH databases. Quantitative and qualitative results show that the proposed EMD based method caters improved results for de-noising and baseline wander removal.
\end{abstract}

Keywords: Electrocardiogram (ECG), Baseline Wander, Empirical mode decomposition (EMD), Morphological filter, Structuring element.

\section{INTRODUCTION}

Electrocardiogram (ECG) signals are one of the well-known biomedical signals. Given their nature, they, bring forward a number of challenges during their registration, processing, and analysis. Characteristic properties of biomedical signals include their non-stationarity, noise sensitivity, and variability among individuals. ECG signals show all these properties. In particular, a highly undesirable aspect of these signals is their high sensitivity to various types of noise. The recorded ECG signals are always affected by noise. The main sources of noise include: variations of the bodyelectrode impedance arising from physio-chemical processes present in this region as well as the movement of the patient. These phenomena cause a so-called effect of a baseline wandering. These are low frequency distortions [1, 2]. Adjustments of a bilateral position of heart and the electrodes caused by respiratory movements of a patient. This rises in some slow-changing disturbances. Contraction of skeletal muscles caused by the movement of patient or the improper temperature of the environment. This also creates muscle disturbances. Due to broad spectrum of frequencies it is very difficult to deal with the frequencies and overlaps with the ECG signal frequency [3]. Interference of electromagnetic nature is often known as power line interferences [4]. High power devices may cause some disturbances such as e.g., diathermy. These noises are of impulse nature [5-6]. By taking into consideration all causes of interferences affecting ECG signals, their list includes: 1) power line interference, 2) electrode contact noise, 3) motion artefacts, 4) EMG noise, 5) instrumentation noise etc. Each of these noise is shown in ideal block diagram in Fig. 1. Thus filtering process is the obvious requirement for accurate diagnosis and removal of most of the constituent noise while it must ensures no loss of vital morphological information of the ECG signal. Baseline wander is usually caused by respiration or movement of the patient. It is one of the most important noise of ECG and appears as low frequency artefact. This artefact is generally found during stress ECG and also ECG signals taken employing holter monitors [7]. 


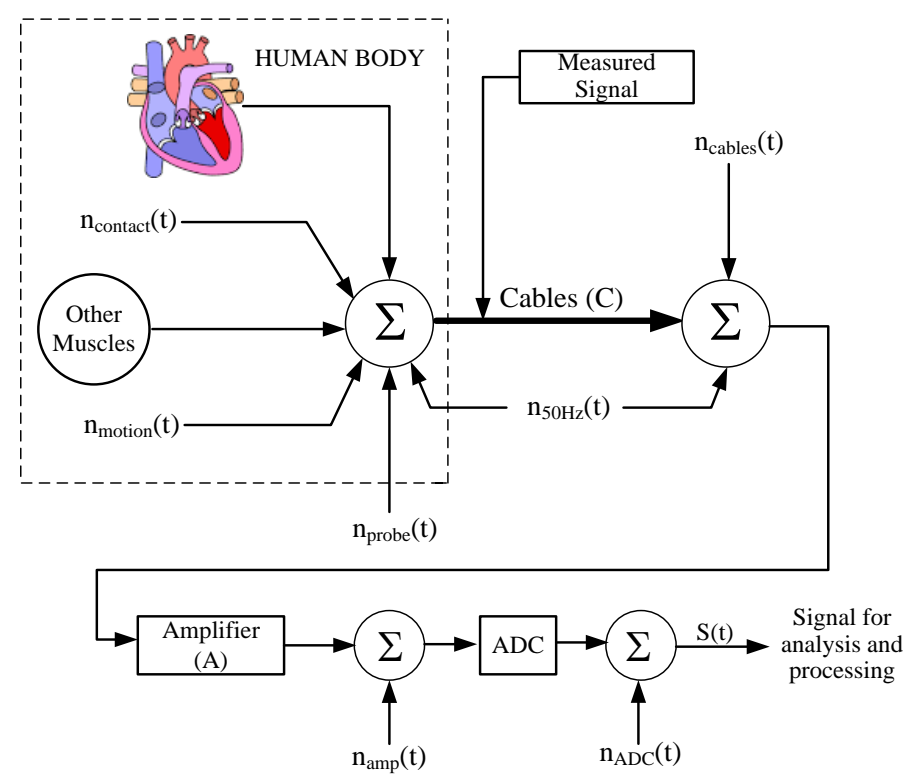

Figure 1. Principal noise sources in ECG (Block diagram).

Many approaches have been reported in the literature for denoising of baseline wander from ECG. Initially window based filters have been used for the removal of this artefact [8], but the ripples are introduced in pass and stop bands, leading errors of morphology. Adaptive algorithms [9-12] are found to not be very effective for noise cancellation. This is due to the fact that the benchmark signal is not correlated certainly with the noise components present in the fundamental input. The choice of membership function becomes constrain for use of fuzzy logic based de-noising [13]. The use of wavelets [1417], Independent Component Analysis (ICA) [18] for baseline wander removal was found to be not satisfactory. EMD [1920] was also used, but the EMD has also the low de-noising capability. Moreover these algorithms did not delineate a definite mechanism for selecting the number of intrinsic mode function (IMF) to be considered. Morphological filters were also used [21-22] however the chosen structuring element was not matched properly with ECG morphology. Morphological operator with linear structuring element has been used in some literature, nevertheless which is bounded to neonatal signals only [23]. Another method based on average of opening and closing operations, the elimination of QRS and then $\mathrm{P}$ and $\mathrm{T}$ waves is more authentic, nevertheless the pulse width has not been fixed [24].

The above discussed methods have been tested widely, but their ability in elimination of baseline wander and impulse noise has been poor. The proposed algorithm achieves denoising in different stages, initially the EMD of baseline drifted impulse noise corrupted ECG signal is performed and a fixed proportion of the total IMFs are considered for next stage of correction. This signal is then applied to a three-step morphological filter dependent on non-flat and flat structuring elements. The performance of the proposed algorithm is demonstrated over few ECG records from the MIT-BIH Arrhythmia Database. Both the quantitative and qualitative analyses are carried out for the noise corrupted ECG signals. The studies show that the proposed method gives improved performance for ECG signal de-noising and BW (baseline wander) removal.

This paper is outlined into six sections. In Section 2 a brief review of EMD, and morphological filter is presented. A complete overview of proposed algorithm of de-noising and baseline wander removal are explained in Section 3. Section 4 illustrates the experimental procedures carried out using proposed algorithm. Section 5 shows the results and the performances of proposed method. Finally conclusions are given in Section 6.

\section{OPERATIONS PERFORMED}

The Empirical mode decomposition (EMD) was proposed by Huang et al. [25] as a device to decompose adaptively a time series into a number of components known as IMFs. IMFs are basically amplitude modulated and frequency modulated components present in any time series. It can deal with nonstationary and nonlinear signals, such as biomedical signals. Some classical data analysis approaches, like Fourier, wavelet-based methods, require some predefined basis functions to represent a signal. EMD is perceptive, direct method, with a posteriori-defined basis, from the decomposition method, and relies on fully data-driven system.

All the intrinsic mode functions (IMFs) can be obtained by decomposing the signal by the use of EMD. An IMF is defined as a function with the number of zero-crossings must either equal or differ at most by one, with its envelopes, as characterized by all the local maxima and minima, being symmetrical with respect to zero axis. An IMF represents a simple oscillatory mode as an equivalent to the simple harmonic function used in Fourier analysis.

Given a time series $x(t)$, the starting point of the EMD is the identification of all the local extrema. Then all the local maxima are connected by a cubic spline line as the upper envelope $E_{u}(t)$. Likewise, the same procedure is repeated for all the local minima to produce lower envelope $E_{l}(t)$. The 
upper and lower envelopes should cover all the extrema between them. Their mean is designated as $m_{l}(t)$ and is given by $m_{1}(t)=\left[E_{u}(t)+E_{l}(t)\right] / 2$ and it is subtracted from the time series. Thus the first proto-IMF $h_{l}(t)$ achieved as

$h_{1}(t)=x(t)-m_{1}(t)$

The said IMF extraction procedure is referred to as sifting process. Since h1(t) still contains numerous maxima and minima in between zero crossings, the h1(t) is again sifted. This operation is practiced repetitively to the proto-IMF $\mathrm{h} 1(\mathrm{t})$ until the first IMF $\mathrm{c} 1(\mathrm{t})$ is obtained, which satisfies IMF condition. A few stoppage criteria are used to terminate the sifting process. The frequently used stoppage criterion determined by using a Cauchy type of convergence test, which requires the normalized squared difference between two successive sifting operations defined as [26].

$$
S D_{k}=\frac{\sum_{t=0}^{T}\left|h_{k-1}(t)-h_{k}(t)\right|^{2}}{\sum_{t=0}^{T} h_{k-1}^{2}}
$$

When the $S D_{k}$ is lesser than a threshold, the sifting process will stop and the first IMF $c_{l}(t)$ is found, which is given as

$$
r_{1}(t)=x(t)-c_{1}(t)
$$

Since the residue $r_{l}(t)$ still contain some suitable information, it is treated as the new signal and subjected to the same sifting process as described above to achieve

$$
\begin{array}{ll}
r_{2}(t)=r_{1}(t)-c_{2}(t) \\
\cdot \\
\cdot \\
\cdot \vec{r} \cdot r_{N}(t)=r_{N-1}(t)-c_{N}(t)
\end{array}
$$

The sifting process can be terminated finally when the residue $r_{n}(t)$ turns either into a constant or a monotonic function from which no more IMFs can be extracted. Adding the equations (3) and (4) gives the EMD of the original time series:

$$
x(t)=\sum_{n=1}^{N} c_{n}(t)+r_{N}(t)
$$

Thus, a decomposition of the signal into $N$ IMFs is achieved, and a residue $r_{N}(t)$ is obtained. For convenience $c_{n}(t)$ is referred as the $n$ th-order IMF. By this convention, lower-order IMFs hold fast oscillation modes, at the same time higherorder IMFs commonly represent slow oscillation modes. In time domain analysis method, lower-order IMFs and higherorder IMFs can be interpreted as fine and coarse scales, respectively. The residue itself can also be considered as IMF.

Morphology, commonly denotes the branch of biology that deals with the form and the structure of animals and plants. Mathematical morphological filtering is a non-linear transformation technique primarily used for local modification of geometrical features of a signal and mostly deals with the mathematical theory of describing shapes using sets. In signal processing, mathematical morphology is used to investigate the interaction between a geometric structure and a certain chosen structuring element using the basic operations of erosion and dilation. It is the effective nonlinear signal processing method and can keep the shape information of a signal good. One more complex morphological operation can be performed with the help of erosion and dilation. The selection of structuring element is the most important factor of these filters. The information from the nonlinear signal can be extracted by varying the shape and size of the SE (structuring element). The shifting of local minima of the signal inside the structuring element or mask is known as erosion of the signal. Similarly movement of local maxima of a signal inside the structuring element is known as dilation. As ECG is a onedimensional signal, the dilation and erosion of onedimensional signal can be defined as follows:

Dilation:

$$
(f \oplus g)(x)=\max _{s}\left\{f(x-s)+g(s) \mid(x-s) \in \mathscr{D}_{f}, s \in \mathscr{D}_{g}\right\}
$$

Erosion:

$$
(f \ominus g)(x)=\min _{s}\left\{f(x+s)-g(s) \mid(x+s) \in \mathscr{D}_{f}, s \in \mathscr{D}_{g}\right\}
$$

where, $\mathscr{D}_{f}, \mathscr{D}_{g}$ are the definition domains of $f$ and $g$, respectively. $f$ is signal under consideration and $g$ is the structuring element. Dilation fills small gap of the signal which was corrupted by noise and perform smoothing. It has also side effect, that the signal boundary gets expanded. Similarly erosion eliminates any extrusion present in the signal. Due to presence of noise these extrusions resembled as a part of the signal. But the aftereffect is that the signal boundary gets reduced or the signal is shrinked. There are more advanced operations and filtering techniques which are based on these basic morphological operators. Here in this work we use two significant operations known as opening and closing, which are derived from the erosion and dilation operations.

Opening: Opening is an operator that performs dilation on a signal eroded by the same structuring element. The definition is given as follows:

$$
f \circ g=(f \ominus g) \oplus g
$$

Closing: It is defined as dilation followed by erosion by the same structuring element. It is given by:

$$
f \bullet g=(f \oplus g) \ominus g
$$

The important property of opening and closing is that they can remove sharp peaks and valleys respectively. These operators can be used to detect peaks and valleys in the signal.

Here the de-noising has been done and is based on the principle of rejection of higher order IMF which is achieved from EMD of the baseline wander and noise corrupted signal, use of morphological filter for extraction of QRS complex removed waveform, low frequency baseline estimation, 
subtraction of estimated baseline from the original signal, thereby reducing the presence of baseline from the signal. The baseline corrected signal again passed through another set of morphological filter for impulse noise suppression.

\section{BASELINE CORRECTION AND NOISE SUPPRESSION}

In this work, proposed algorithm was applied to real time ECG data for removal of baseline wander noise and as well as impulsive noise. The algorithm consists of breaking down the noise corrupted signal into IMFs and then passing through morphological filters as shown in block diagram shown in Fig. 3.

First step:

Given noisy signal is decomposed into $N$ IMFs using EMD as shown in Equation 5. Based on the experimental observations and Abdel-Ouahab Boudraa et al. [27], the time series of higher order IMFs to be discarded to obtain partially filtered ECG signal. Higher order IMFs contain slow changing frequencies, which contribute baseline. Based on simulation results on experimental basis, $24 \%$ of higher IMFs are discarded in this present study.

$$
p=\operatorname{round}(0.24 \times N)
$$

Thus the partially filtered ECG signal which was reconstructed from $(N-p)$ IMFs becomes

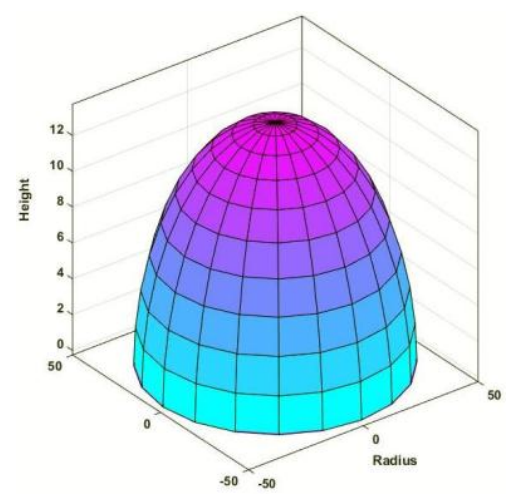

Figure 2. Ball shaped non-flat structuring element (a) $\mathrm{S}_{1}$ : radius 49 and height 27 , (b) $\mathrm{S}_{2}$ : radius 30 and height

10

Third step: As per the proposed algorithm, signal obtained after first phase of morphological operation by non-flat structuring elements, is used to perform two sets of operation. In first stage opening-closing is performed, where as in second stage closing-opening is performed with the flat structuring elements to eliminate QRS complex from the ECG signal. Based on the average length of QRS complex, a flat structuring elements of length $0.12 \times F_{s} \quad\left(F_{s}\right.$ : sampling frequency of ECG data) can be used. QRS complex removed ECG time series is given by

$y(t)=\left[x(t) \circ S_{3} \bullet S_{3}+x(t) \bullet S_{3} \circ S_{3}\right] / 2$
$x(t)=\sum_{n=1}^{N-p} c_{n}(t)$

The partial filtering is done to assure that no shape related information is lost.

\section{Second step:}

In the proposed algorithm, both flat and non-flat structuring elements for the morphological filters are used. An important aspect in baseline removal and noise elimination is the selection of optimum size of the structuring element. Inaccurate selection of structuring element may distort the adjacent wave in the ECG signal. Hence, the size of structuring element should be greater than the width of the ECG characteristic wave signal [28]. Flat structuring elements frequently causes distortion due to overlap of low frequency (ST) segment with the baseline wandering, resulting the relevant information may be lost [29]. Thus, a combination of flat and non-flat structuring element is proposed in this work for pre-processing better extraction of characteristic wave for baseline correction without introducing any distortion in ECG waveform. A ball-shaped structuring elements as shown in Fig. 2 are used with the proposed algorithm for pre-processing of ECG signal prior to extraction of QRS complex removed signal. The dimensions of the structuring element are determined experimentally using the performance evaluation parameters. It can be analysed as non-linear filters which polishes the contours of input ECG time series.

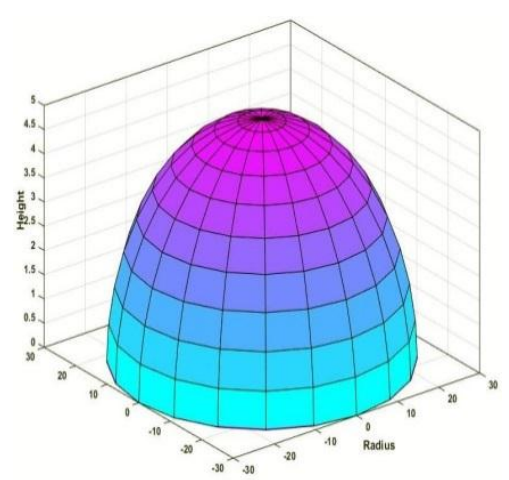

where, $S_{3}$ is the flat structuring element based on average length of QRS complex.

Fourth step:

In this stage it is to remove the $\mathrm{P}$ and $\mathrm{T}$ wave from $y(t)$. So, the QRS complex removed ECG signal is passed through a second morphological operation. Therefore compared with structuring element $S_{3}$, the length of the $S_{4}$ should exceed the length of these waves. Based on average ST wave time interval, the length of second flat structuring element is chosen and is given by $0.3 \times F_{s}\left(F_{s}\right.$ : sampling frequency of ECG data). 
$z(t)=\left[y(t) \circ S_{4} \bullet S_{4} \circ S_{4}+y(t) \bullet S_{4} \circ S_{4} \bullet S_{4}\right] / 2(13)$

Now the signal $z(t)$ does not contain any of the morphological ECG segments. It is an estimate of the total remaining baseline wander present in the original time series. This baseline noise obtained in step four is subtracted from raw and noise corrupted ECG signal to get baseline wander free ECG signal and is given by

$$
p(t)=x(t)-z(t)
$$

Fifth step:

ECG signal is also corrupted with high frequency impulse noise. During removal of baseline wander, some of the impulse noise is removed. However, for further noise elimination, the signal obtained after baseline correction $p(t)$ is made input to the proposed noise suppression algorithm, which involves top-hat and bottom-hat transformation using structuring element of optimum shape and size. Top-hat and bottom-hat transformation using structuring element $\left(S_{5}\right)$ returns high frequency noisy component which corrupts ECG signals and is subtracted from baseline corrected ECG signal to get de-noised signal.

Top-hat transformation: $T_{\text {hat }}(t)=f-(f \circ g)$

Bottom-hat transformation: $B_{\text {hat }}(t)=(f \bullet g)-t$

After this noise suppression algorithm the estimation of impulsive noise can be obtained and is given by

$$
I_{N}(t)=\left[p(t)-\left\{P(t) \circ S_{5}\right\}\right]-\left[\left\{p(t) \bullet S_{5}\right\}-p(t)\right]
$$

And the final de-noised signal becomes

$$
q(t)=p(t)-I_{N}(t)
$$

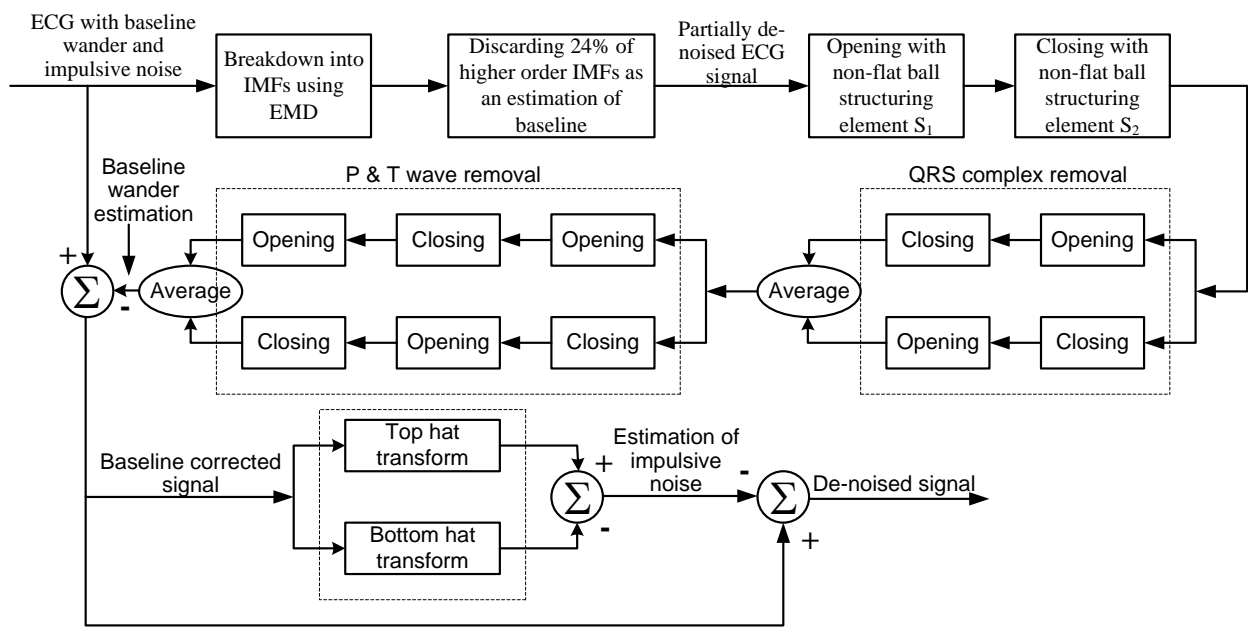

Figure 3. Block diagram of proposed baseline and impulsive noise removal algorithm.

\section{EXPERIMENTAL PROCEDURE}

This algorithm is tested on real time ECG signal. In this simulation studies, test ECG signal is taken from MIT-BIH Arrhythmia Database [30]. The recordings were digitized at 360 samples per second per channel with 11-bit resolution over a $10 \mathrm{mV}$ range. To demonstrate the feasibility of the proposed algorithm, an artificial baseline wander noise is added to the original impulsive noise corrupted test signal taken from MIT-BIH database. The emerged signal is thus referred to as the baseline drifted and impulse noise corrupted signal. The artificial baseline wander is developed from a random signal filtered by a low pass Butterworth filter. The magnitude of the samples of the random signal are uniformly distributed in the range of 0 to $m$, where $m$ is known as amplitude of added artificial baseline wander. In Fig. 4 the dotted line signifies the result of summating an artificial baseline wander (BW) to the impulsive noise corrupted test signal. Whereas the impulsive noise corrupted test signal is depicted by solid line in Fig. 4. The normalised baseline corrected ECG signal is illustrated in Fig. 5, from which it can be seen that the proposed scheme works healthy under the condition of severe baseline drift. It is well known that the test signals are not absolutely baselinefree, thus the normalisation process eliminates both artificial BW and the BW existing in the test signal. Hence, the normalised signal is not identical to the test signal (taken from MIT-BIH database). 


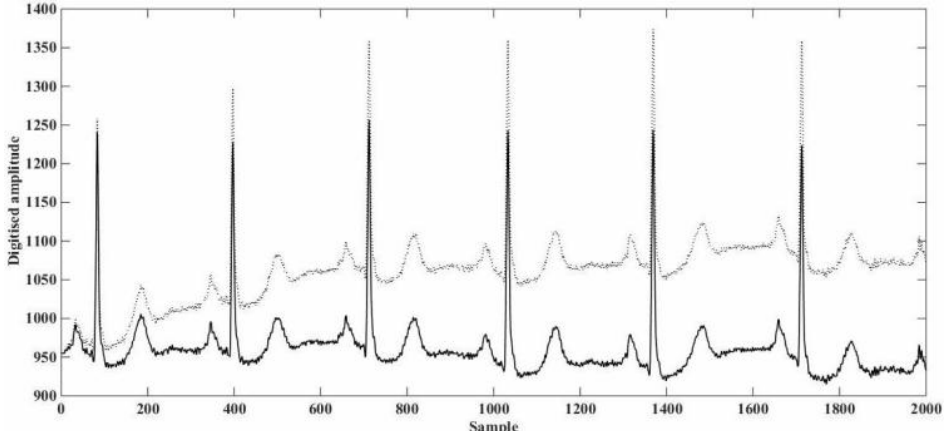

Figure 4. Dotted line: baseline corrupted ECG test signal $(m=300)$, Solid line: ECG test signal taken from MIT-BIH data base.

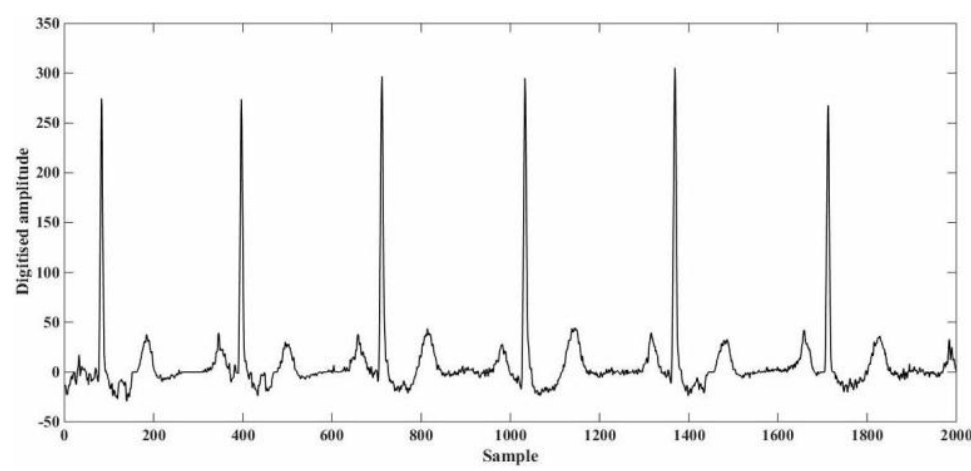

Figure 5. Normalised baseline corrected ECG signal

After elimination of baseline wander, the impulsive noise corrupted normalised signal is passed through impulsive noise suppression algorithm shown in Fig. 3. Fig. 6 shows the ultimate de-noised ECG signal.
The structuring element used for the impulsive noise suppression is compared with the width of the noise component present, hence noise can be removed without sacrificing the signal quality.

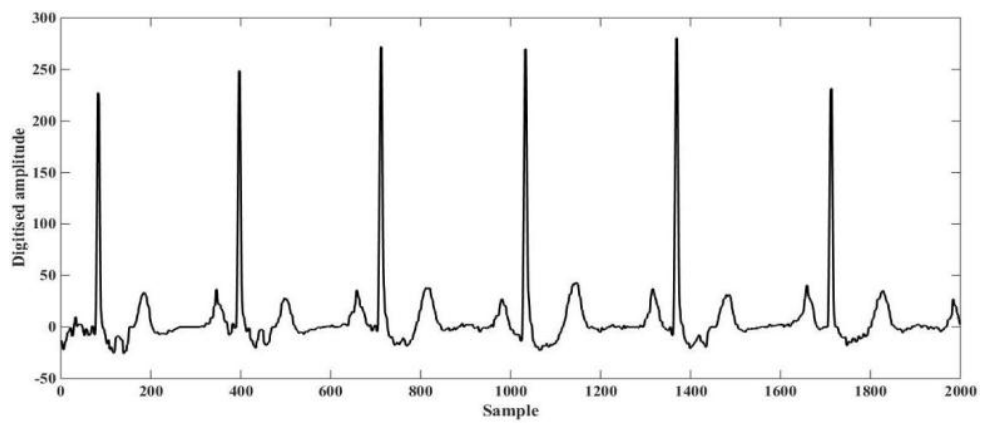

Figure 6. De-noised ECG signal

\section{RESULTS AND PERFORMANCES}

The performance of proposed algorithm evaluated with the help of two qualitative parameters: correlation criterion $(r)$ and signal-to-noise ratio $(S N R)$ in $d B$. Correlation criterion reflects the semblance or linear interdependence between the two signals considered. If the two signals are identical, the value of $r$ is 1 and if the two signals are exactly inverse of each other, then the value of $r$ is -1 . It is defined as $r=\frac{n\left(\sum X Y\right)-\left(\sum X\right)\left(\sum Y\right)}{\sqrt{\left[n \sum X^{2}-\left(\sum X\right)^{2}\right]\left[n \sum Y^{2}-\left(\sum Y\right)^{2}\right]}}$, where $X$ and $Y$ are the two signals under investigation, $\mathrm{n}$ is the length of the signals. $S N R$ is used to assess the noise level in the ECG signal, thus higher the value of SNR, better is the amount of noise suppression. Therefore $S N R=10 \times \log _{10} \frac{S_{\sigma}}{N_{\sigma}}$, where $S$ and $N$ represent the ECG signal and the baseline wander (BW), respectively. For a given signal $Y, Y_{\sigma}$ can be defined as $Y_{\sigma}=\sum_{n=0}^{L-1}\left[Y(n)-\mu_{Y}\right]^{2}$, where $\mu_{Y}$ and $L$ mean and length of signal $Y$ respectively.

In this present studies, five artificial BWs with amplitudes 
of $100,300,500,700$ and 900 are added respectively with each ECG test signal (record 101, 116 and 205). Tables 1 and 2 list the correlations and signal-to-noise ratios respectively of baseline wander normalised three ECG signals and also compared with Ji's method [7].

Table 1. Comparison of performances of proposed method and Ji's method subject to correlation criterion $(r)$

\begin{tabular}{|c|c|c|c|c|}
\hline \multirow{2}{*}{$\begin{array}{c}\text { Recorded } \\
\text { ECG signal } \\
\text { (MIT-BIH } \\
\text { Arrhythmia } \\
\text { database) }\end{array}$} & \multirow{2}{*}{$\begin{array}{c}\text { Amplitude } \\
\text { of artificial } \\
\text { baseline } \\
\text { wander }\end{array}$} & \multicolumn{3}{|c|}{ Correlation coefficient (r) } \\
\hline & & $\begin{array}{c}\text { Test } \\
\text { ECG } \\
\text { Signal }\end{array}$ & $\begin{array}{c}\text { Ji's } \\
\text { method }\end{array}$ & $\begin{array}{l}\text { Proposed } \\
\text { algorithm }\end{array}$ \\
\hline \multirow{5}{*}{101} & 100 & 0.947 & 0.823 & 0.960 \\
\hline & 300 & 0.631 & 0.643 & 0.757 \\
\hline & 500 & 0.371 & 0.456 & 0.561 \\
\hline & 700 & 0.237 & 0.367 & 0.468 \\
\hline & 900 & 0.139 & 0.250 & 0.407 \\
\hline \multirow{5}{*}{116} & 100 & 0.743 & 0.833 & 0.989 \\
\hline & 300 & 0.651 & 0.840 & 0.962 \\
\hline & 500 & 0.871 & 0.766 & 0.900 \\
\hline & 700 & 0.792 & 0.720 & 0.835 \\
\hline & 900 & 0.695 & 0.704 & 0.756 \\
\hline \multirow{5}{*}{205} & 100 & 0.801 & 0.819 & 0.928 \\
\hline & 300 & 0.703 & 0.780 & 0.897 \\
\hline & 500 & 0.533 & 0.542 & 0.552 \\
\hline & 700 & 0.416 & 0.425 & 0.459 \\
\hline & 900 & 0.354 & 0.389 & 0.412 \\
\hline
\end{tabular}

Table 2. Comparison of Performances of Proposed Method and Ji's Method Subject to Signal-to-Noise Ratio (SNR)

\begin{tabular}{|c|c|c|c|c|}
\hline \multirow{2}{*}{$\begin{array}{c}\text { Recorded } \\
\text { ECG signal } \\
\text { (MIT-BIH } \\
\text { Arrhythmia } \\
\text { database) }\end{array}$} & \multirow{2}{*}{$\begin{array}{c}\text { Amplitude } \\
\text { of artificial } \\
\text { baseline } \\
\text { wander }\end{array}$} & \multicolumn{3}{|c|}{ Signal-to-Noise Ratio (SNR) } \\
\hline & & $\begin{array}{c}\text { Test } \\
\text { ECG } \\
\text { Signal }\end{array}$ & $\begin{array}{c}\text { Ji's } \\
\text { method }\end{array}$ & $\begin{array}{l}\text { Proposed } \\
\text { algorithm }\end{array}$ \\
\hline \multirow{5}{*}{101} & 100 & 4.487 & 10.562 & 25.243 \\
\hline & 300 & -0.077 & 5.019 & 8.514 \\
\hline & 500 & -4.370 & 2.196 & 3.747 \\
\hline & 700 & -6.982 & 1.342 & 2.424 \\
\hline & 900 & -9.490 & 0.634 & 1.698 \\
\hline \multirow{5}{*}{116} & 100 & 4.815 & 11.164 & 38.403 \\
\hline & 300 & 4.530 & 11.054 & 25.908 \\
\hline & 500 & 2.700 & 8.158 & 16.559 \\
\hline & 700 & 1.423 & 6.815 & 11.916 \\
\hline & 900 & -0.323 & 4.964 & 8.431 \\
\hline \multirow{5}{*}{205} & 100 & 3.475 & 9.839 & 19.772 \\
\hline & 300 & -2.013 & 3.877 & 6.719 \\
\hline & 500 & -5.720 & 1.827 & 3.533 \\
\hline & 700 & -8.338 & 1.373 & 2.219 \\
\hline & 900 & -10.543 & 0.489 & 1.671 \\
\hline
\end{tabular}

From the above set of tabulations it can be observed that in all instances the proposed algorithm improves correlation coefficient (r) and signal-to-noise ratio (SNR) significantly.
Larger values of correlation coefficient illustrates better similarity between the signals of consideration. Moreover, higher values of $S N R$ obtained in the present work depicts exceptional noise suppression in comparison to others algorithms.

The performance of the proposed algorithm is also compared with EMD and MM based method as proposed by Ji et al. [7]. The added artificial BWs are the identical as used in Ji's method. The correlation coefficients and signalto-noise ratios of the normalised signals achieved in Ji's simulation are also enlisted in Tables 1 and 2, respectively. From the Tables it is observed that in all cases proposed algorithm accomplishes better results than Ji's method, under both correlation coefficient and SNR benchmarks. Beside the baseline elimination, this proposed algorithm also eliminates the high frequency impulse noise from the ECG test signal with a little attenuation of the $\mathrm{R}$ peaks, which is no doubt more advantageous in comparison with the Ji's method. Owing to the complex EMD decomposition procedure, the computation time is more or less same for both the algorithms.

\section{CONCLUSION}

A novel method based on empirical mode decomposition and morphological filter for ECG baseline correction and high frequency impulse noise suppression is presented. The original ECG signal is decomposed into series of IMFs by using EMD. The algorithm formulated in this paper is based on the fact that the higher order IMFs have strong BW components merged with the signal. So some portion of higher order IMFs are discarded in this method. In this work, an improved algorithm using both flat and non-flat structuring element is proposed to eliminate baseline drift and high frequency impulse noise from the ECG signal. The non-flat structuring element possesses advantage over flat structuring element as it minimizes the distortion produced by flat structuring element during baseline line correction and noise removal. A number of simulations are carried out to investigate the validity of the approach. Simulation results indicate that the proposed method is surely capable to eliminate $\mathrm{BW}$ and impulse noise while protecting the waveform morphological information.

\section{REFERENCES}

[1] Wiese, S.R., Anheier, P., Connemara, R.D., Mollner, A.T., Neils, T.F., Khan, J.A., Webster, J.G.: Electrocardiographic motion artifact versus electrode impedance. IEEE Trans. Biomed. Eng. 52(1), 136-139 (2005).

[2] Pawar, T., Chaudhuri, S., Duttagupta, S.P.: Body movement activity recognition for ambulatory cardiac monitoring. IEEE Trans. Biomed. Eng. 54(5), 874-883 (2007b).

[3] Fernandez-Chimeno, M., Quilez, M., Silva, F.: Understanding electrosurgical unit perturbations in order to address hospital operating room electromagnetic compatibility. IEEE Trans. 
Biomed. Eng. 53(6), 1205-1207 (2006).

[4] Bruce, E.N.: Biomedical Signal Processing and Signal Modeling. Wiley, New York (2000).

[5] Santopietro, R.F.: The origin and characterization of the primary signal, noise, and interference sources in the high frequency electrocardiogram. Proc. IEEE 65(5), 707-713 (1977).

[6] Fernandez-Chimeno, M., Quilez, M., Silva, F.: Understanding electrosurgical unit perturbations in order to address hospital operating room electromagnetic compatibility. IEEE Trans. Biomed. Eng. 53(6), 1205-1207 (2006).

[7] Ji, T.Y., Lu, Z., Wu, Q.H., and Ji, Z.: 'Baseline normalization of ECG signals using empirical mode decomposition and mathematical morphology', Electron. Letters. 2008, 44, (2), pp. 82-83.

[8] SK. Jagtap, MS. Chavan, RC. Wagvekar and MD. Uplane, "Application of the digital filter for noise reduction in electrocardiogram". Journal of Instrumentation, vol .40, no. 2, pp. 83-86, June 2010.

[9] V. Almenar, A. Albiol, A new adaptive scheme for ECG enhancement, Signal Process. 75 (3) (1999) 253-263.

[10] Dr. K. L. Yadav and Sachin Singh, "Performance evaluation of different adaptive filters for ECG signal processing", International Journal On Computer Science and Engineering, vol. 40, no. 5, pp. 1880-1883, 2010.

[11] Muhammad ZiaUrRahman, Rafi Ahamed Shaik and D.V.RamaKoti Roddy, "Efficient sign based normalized adaptive filtering techniques for cancelation of artifacts in ECG signals: Application to wireless biotelemetry", Journal of signal processing, vol. 91, no. 2, pp. 225-239, February 2011.

[12] Ching-Haur Chang ,Kang-Ming Chang ,and Hsien$\mathrm{Ju} \mathrm{Ko,} \mathrm{"Cancellation} \mathrm{of} \mathrm{high} \mathrm{frequency} \mathrm{noise} \mathrm{in}$ ECG signals using Adaptive filter without external reference", Proceedings of International Conference on Biomedical Engineering and Informatics, pp. 787-790, Yantai, October 2010.

[13] C.Y-F Ho, B.W.-K, Ling, T.R-L. Wong, A.Y.-P chan. P.-K.-S.Tam, "Fuzzy rule based multiwavelet Electrocardiogm (ECG) denoising”, Fuzzy Systems, IEEE international Conference,pp.no 1064-1068, 2008.

[14] P.E. Tikkanen, Nonlinear wavelet and wavelet packet denoising of electrocardiogram signal, Biol. Cybern. 80 (4) (1999) 259-267.

[15] C.Y.-F. Ho, B.W.-K. Ling, T.P.-L. Wong, A.Y.-P.
Chan, P.K.-S. Tam, Fuzzy multiwavelet denoising on ECG signal, Electron. Lett. 39 (16) (2003) 1163-1164.

[16] E. Ercelebi, Electrocardiogram signals de-noising using lifting-based discrete wavelet transform, Comput. Biol. Med. 34 (6) (2004) 479-493.

[17] S. Poornachandra, N. Kumaravel, Hyper-trim shrinkage for denoising of ECG signal, Digital Signal Process. 15 (3) (2005) 317-327.

[18] A.K. Barros, A. Mansour, N. Ohnishi, Removing artifacts from electrocardiographic signals using independent components analysis, Neurocomputing 22 (1998) 173-186.

[19] A.O Boudraa,J-C cexus,"EMD Based signal filtering", IEEE Transactions on instrumentation and measurement, vol 56,no. 6,pp 2196-2202, 2007.

[20] Na Pan, vai MingI, Mai Peng Un and Pun Sio hang," Accurate removal of baseline wander in ECG using EMD”, proceedings of NFSI \& ICFBI 2007 China, pg no 177-180.

[21] Chee Hung Henry Chu and Edward J Delp, "Impulsive noise suppression and background normalization of ECG signals using morphological operators", IEEE transactions in biomedical engineering, vol 36,no.2,Feburary, 1989,pg no. 262-273.

[22] Seyfullah Halit OGUZ and Muka Hakan Asyali, "A morphology based algorithm for baseline wander elimination in ECG records, International Biomedical Engineering days, 1992, pg no. 157-160.

[23] P Sun, Q.H Wu, A.M Weindling, A Finklestein and $\mathrm{K}$ Ibrahim, "An improved morphological approach to background normalization of ECG signals".IEEE trnasactions on biomedical engineering, VOl 50, No1. January 2003, pg no 117-121.

[24] Zhongguo Liu, Jinliang Wang and Boqiang Liu, "ECG signal denoising based on morphological filtering", $5^{\text {th }}$ International conference on Bioinformatics and Biomedical Engineering, 2011.

[25] N.E. Huang, Z. Shen, S.R. Long, M.C. Wu, H.H. Shih, Q. Zheng, N.- C. Yen, C.C. Tung, H.H. Liu, The empirical mode decomposition and Hilbert spectrum for nonlinear and non-stationary time series analysis, Proc. R. Soc. London 454 (1998) 903-995.

[26] N. Huang, N.O. Attoh-Okine, in: N. Huang, N.O. Attoh-Okine (Eds.), The Hilbert-Huang Transform in Engineering, CRC Press, Boca Raton, FL, 2005.

[27] A.O Boudraa, J-C cexus,"EMD Based signal filtering", IEEE Transactions on instrumentation 
International Journal of Applied Engineering Research ISSN 0973-4562 Volume 12, Number 10 (2017) pp. 2329-2337 (C) Research India Publications. https://dx.doi.org/10.37622/IJAER/12.10.2017.2329-2337

and measurement, vol 56,no. 6,pp 2196-2202, 2007.

[28] Chee-Hung Henry Chu and E. J. Delp, "Electrocardiogram Signal Processing by Morphological Operators," Proc. of the Conference on Computers in Cardiology, Washington DC, pp. 153-156, September 1988.

[29] Yuan Gu, Gang Zheng and Min Dai, "A
Morphology Algorithm Based on Two Dimensional Flat Structuring Element on ECG Baseline Wander Elimination," Proc. of the Conference on Computing in Cardiology, Hangzhou, pp. 817-820, September 2011.

[30] https://www.physionet.org/physiobank/database/mi $\mathrm{tdb}$ 